BABELAO 4 (2015), p. 135-148

(C) ABELAO (Belgium)

\title{
La division du temps dans l'Église primitive : une structure septennale dans l'évangile selon Marc
}

Par

\section{Christian-Bernard Amphoux}

$C P A F$, Université d'Aix-Marseille

\begin{abstract}
A u temple de Jérusalem, les années étaient comptées en cycle de sept années, la septième étant une année dite sabbatique. Et pour déterminer celle-ci, on dispose d'un repère en $1 \mathrm{M}$ 6,49.53, selon lequel l'année -164-163 fut une année sabbatique ${ }^{1}$ : on peut donc établir tout le calendrier du temple et dater les années sabbatiques. Ainsi, -604, l'année où la Judée est intégrée à l'empire babylonien, fut une année sabbatique ; tandis que -586, l'année de la prise de Jérusalem par le même roi de Babylone, Nabuchodonosor, sera une quatrième année de cycle sabbatique, une année funeste. En avançant dans le temps, on arrive à celui de Jésus : les années 25 et 32 sont sabbatiques, elles encadrent le ministère de Jésus qui va du printemps 28 à la Pâque de l'an 30. Et la question se pose de savoir comment les chrétiens
\end{abstract}

1 Année où Judas Maccabée purifie le temple et y rétablit le culte, après la profanation d'Antiochos IV Epiphane, trois ans plus tôt, qui reste dans la

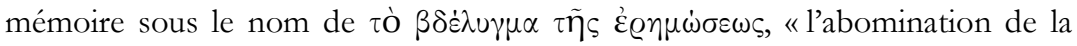
désolation ». Rappelons que l'année civile commence alors le $1^{\mathrm{er}}$ avril. 
ont compté le temps, au début de leur histoire : ont-ils utilisé un calendrier cyclique et sur quelle base?

On ne disposait, jusqu'à présent, d'aucun indice pour traiter un tel sujet, si bien que la question ne se posait pas. Mais un indice existe dans l'évangile de Marc, et je l'ai découvert il y a quelques années, au hasard d'une analyse littéraire dont la première partie a été publiée ${ }^{2}$. Selon cet indice, les chrétiens ont continué à compter le temps par cycles de sept années au moins jusqu'en 70, mais avec un décalage par rapport au calendrier du temple, comme nous allons le voir. Cet indice montre à la fois la judéité de la première génération chrétienne et son souci d'autonomie par rapport au judaïsme du temple de Jérusalem. Il s'agit donc de préciser l'indice en question et de fonder les conclusions que je viens d'indiquer.

\section{La première tradition narrative de Marc}

L'étude parue en 1997 (n. 2) arrive à la conclusion que Marc n'est pas écrit d'un seul tenant, mais que cet évangile est la réunion de deux traditions narratives, l'une écrite avant 70 et l'autre dans les années 80-90, la réunion se faisant vers 120 ; et une deuxième étude parue en 2003 montre que, vers 120, la rédaction finale coïncide avec l'édition des quatre évangiles dans laquelle Marc figure en quatrième position ${ }^{3}$. Ces résultats s'éloignent de la théorie dite des deux sources, qui voit dans Marc la tradition narrative utilisée par Matthieu et Luc, donc un évangile rédigé avant les autres ; il faut donc rappeler ce qui fonde cette analyse.

\section{Deux traditions narratives}

La comparaison des évangiles synoptiques met en évidence que, dans l'ensemble, les épisodes du ministère sont les mêmes dans les trois livres; mais avec une difficulté : bon nombre de ces épisodes sont disposés dans le même ordre, tandis que d'autres ne le sont pas ou le sont seulement dans deux des évangiles. La solution traditionnelle a été de dire qu'il s'agissait de thèmes de prédication $^{4}$ et non de faits ou d'événements du ministère; mais une

${ }^{2}$ C.-B. Amphoux, «Quelques remarques sur la formation, le genre littéraire et la composition de l'évangile selon Marc », Filologia neotestamentaria 10/12 (1997), p. 5-34.

${ }^{3}$ C.-B. AmpHoux, « Marc comme quatrième évangile », C.-B. AmpHOuX J. K. ElLiOTt (éd.), Le texte du Nouveau Testament au début du christianisme / The Text of the New Testament in Early Christianity, colloque de Lille, juillet 2000, HTB 6, Lausanne, Le Zèbre, 2003, p. 329-347.

${ }^{4}$ C'est le cas de Richard Simon, dans son Histoire critique du texte du NT (Rotterdam, 1689) ; puis G. E. Lessing (1774) fait l'hypothèse que les synoptiques utilisent comme source un évangile primitif en araméen dans lequel les récits sont détachés, donc à une place libre. Le propos de Lessing marque le début de l'histoire de la rédaction des évangiles; il est complété par celui de F. Schleiermacher (1832) qui distingue deux sources, l'une narrative (Marc) et l'autre de paroles (Matthieu), en se fondant sur le témoignage de Papias (voir L. VAGANAY, Le problème synoptique, Paris - Tournai, 1952, p. 2-4). 
observation nouvelle oriente vers une autre solution : si l'on sépare les épisodes communs et disposés dans le même ordre dans les trois évangiles, on constate que cet ensemble commun a une organisation régulière qui ne s'explique que par une source spécifique; autrement dit, l'ensemble commun des synoptiques a comme origine une tradition propre, et celle-ci raconte l'histoire du ministère de Jésus, de son baptême, au printemps de l'an 28 , à sa mort, la veille de la Pâque de l'an 30. Les autres épisodes viennent donc d'une tradition distincte, une au moins, à laquelle nous allons nous intéresser plus particulièrement.

Une source suffit à rendre compte des autres épisodes de Marc, grâce à un témoignage récemment retrouvé dans une lettre de Clément d'Alexandrie ou d'un de ses contemporains, soit du début du $\mathrm{III}^{\mathrm{e}}$ siècle $^{5}$. D'après ce témoignage, l'évangile de Marc a été écrit en deux temps, d'abord à Rome, du vivant de Pierre donc avant 65, si Pierre est bien mort lors de la persécution de juillet 64, consécutive à l'incendie de Rome ${ }^{6}-$, comprenant des " actes du Seigneur ", donc des récits; puis à Alexandrie, après le martyre de Pierre, cette seconde partie amplifiant la première par de nouveaux récits empruntés à des «mémoires » de Pierre et par des paroles de Jésus destinées à conduire l'auditeur autorisé « dans le sanctuaire inaccessible de la vérité cachée par sept voiles ». Or, le reliquat de Marc, une fois mis de côté l'ensemble commun, reproduit cette tripartition: (1) douze épisodes sont communs à Marc et Luc et se trouvent le plus souvent aussi dans Matthieu, mais la place n'est jamais la même dans les trois livres ; (2) une séquence narrative en cinq épisodes s'y ajoute, étant commune à Marc et Matthieu; (3) sept paroles de Jésus interviennent dans des épisodes communs à Marc et Matthieu et se trouvent également dans l'Evangile selon Thomas et, en partie seulement, dans la collection de paroles de Luc (10,23-18,14), ajoutant dans Marc une réflexion à la fois stratégique et théologique.

La conclusion que nous en avons tirée est que cet ensemble tripartite forme la première tradition narrative de Marc, rédigée en deux temps au cours des années 60, et correspond au livre dont témoigne la lettre de Clément. L'ensemble commun des synoptiques représente une deuxième tradition narrative, rédigée après 70. Mais une nouvelle difficulté a surgi : les deux histoires, celle des douze épisodes de la première tradition narrative et celle de la deuxième tradition narrative, semblent raconter l'histoire du ministère de Jésus ; or, elles n'ont presque rien en commun; et cela

${ }^{5}$ M. SMITH, Clement of Alexandrie and a Secret Gospel of Mark, Cambridge (Mass.), 1973 ; trad. française par J.-D. KAESTLI, dans Ecrits apocryphes chrétiens, vol. 1, La Pléiade, Paris, 1997, p. 63-69. La date de la lettre me paraît garantie par le mélange des types de texte des citations de Marc, qui correspond à celui des citations faites par Clément d'Alexandrie.

${ }^{6}$ D'après Tacite (Annales XV, 44), les chrétiens furent alors transformés en torches vivantes. 
pose la question: l'une des deux traditions narratives raconte-telle autre chose que le ministère de Jésus?

\section{Le " Marc Romain »}

Convenons d'appeler la première partie de la première tradition narrative, celle que nous faisons coïncider avec la rédaction de Marc à Rome avant la mort de Pierre, de «Marc romain». Cette série d'épisodes communs à Marc et Luc comprend les passages suivants :

1) Mc 1,21-39 Jésus à Capharnaüm

2) Mc 3,7-19 l'appel des Douze

3) Mc 3,31-35 relégation de la famille

4) Mc 4,21-25 les paroles de Jésus

5) Mc 4,35-41 la tempête apaisée

6) Mc 5,1-20 l'exorcisme d'un démoniaque

7) Mc 5,21-43 la guérison de deux femmes

8) Mc 6,1-6 Jésus dans sa patrie

9) Mc 6,7-13 l'envoi en mission des disciples

10) Mc $9,38-50$ un incident en mission

11) Mc 12,41-44 l'aumône de la veuve

12) Mc 14,3-9 l'onction mortuaire de Béthanie

A première vue, ces épisodes semblent indépendants les uns des autres, pour la plupart, si bien qu'ils paraissent justifier la thèse de récits détachés qui expliquerait que leur place ne soit pas constante, d'un évangile à l'autre. Mais un détail m'a soudain intrigué.

Dans l'épisode de l'appel des Douze, la liste des régions qui figure en Mc 3,7-8 se lit ainsi dans le texte « occidental » (D) ${ }^{7}$ :

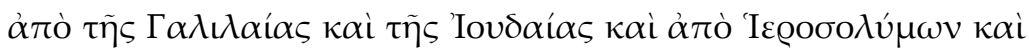

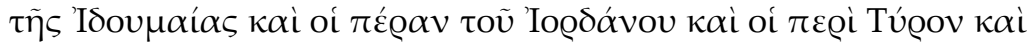
oí $\pi \varepsilon @ i ~ \Sigma เ \delta \tilde{\omega} v \alpha$, «venant de la Galilée et la Judée et venant de Jérusalem et l'Idumée et ceux au-delà du Jourdain et ceux autour de Tyr et ceux autour de Sidon...»

Spontanément, on repère une opposition entre la Galilée et la Judée, d'une part, et les quatre régions limitrophes, de l'autre, avec Jérusalem comme point central ; mais, les prépositions imposent une autre lecture, qui s'estompe dans les autres types de texte: deux couples (venant de la Galilée et la Judée / et venant de Jérusalem et l'Idumée) s'opposent à un troisième (ceux autour de Tyr et ceux autour de Sidon), de part et d'autre d'une région centrale (ceux au-delà du Jourdain). Le centre de Jérusalem est alors remplacé par une région, et cette substitution mène à une lecture historique et non plus géographique : au-delà du Jourdain se trouve l'axe rou-

${ }^{7}$ Le texte de ce passage varie d'un manuscrit à l'autre : voir art. cit. (n. 3), p. 340-345. Mais la variante-source, celle à partir de laquelle on peut expliquer l'existence des autres, est clairement ici la leçon « occidentale ». 
tier de Pétra à Damas que l'on emprunte nécessairement pour aller de Jérusalem à Damas; autrement dit, la structure évoque le chemin de Damas, lieu de la conversion de Paul. Avant cette conversion, la communauté primitive est divisée en deux courants représentés par deux couples (disciples Galiléens, Hellénistes Iduméens); après la conversion, la prédication s'étend au monde grec, représenté par les gens de Tyr et de Sidon. En clair, la liste des régions, sous une apparente géographie, propose une lecture historique de la première génération chrétienne, en faisant de la conversion de Paul un événement central. Mais que fait cette allusion à la conversion de Paul dans un récit évangélique ? On comprend que les autres types de texte l'aient ensuite éliminé ; mais que signifie-t-elle dans la leçon qui est la variante-source des autres?

J'ai relu avec cette question l'ensemble des douze épisodes, et un deuxième sens m'est apparu, liant ces épisodes les uns aux autres. En voici un aperçu :

1) Jésus à Capharnaüm. La fin de l'épisode fait allusion à une disparition et une réapparition de Jésus qui fait penser à sa mort et résurrection; dans ces conditions, on peut chercher à voir dans cette hypothèse un résumé du ministère de Jésus et non son seul début.

2) L'appel des Douze. Les régions (Mc 3,7-8), on vient de le voir, forment un sommaire de la première génération chrétienne. La liste des Douze a également un deuxième sens : des trois groupes de quatre apôtres qui la composent ${ }^{8}$, le premier représente la direction primitive de la communauté, le deuxième réunit les protagonistes d'un débat autour des paroles, et le dernier annonce le temps de la gestion de la communauté par Jacques et ses frères, qui est aussi celui de la prédication de Paul'. Les noms propres de l'épisode constituent, en somme, un double sommaire de la première génération chrétienne, développé par les épisodes 3-11.

3-4) Relégation de la famille / les paroles de Jésus. Ces deux courts épisodes s'associent pour faire allusion à la première décision de la communauté primitive, après la mort de Jésus : la mise en place de la direction. Celle-ci est confiée aux disciples et non à la famille de Jésus, qui pouvait prétendre à une succession dynastique, et les

${ }^{8}$ Comparons cette liste des Douze avec les trois autres qui figurent dans le NT (Mt 10,2-4 / Lc 6,14-16 / Ac 1,13) : trois noms ont une place fixe, Simon Pierre, Philippe et Jacques d'Alphée ; l'ordre des autres varie, mais à l'intérieur du même groupe de quatre, qui commence par l'un des noms précédents.

\footnotetext{
${ }^{9}$ Paul est, en effet, présent sous le personnage de Judas Iscariote, à travers les jeux de mots auxquels se prête le surnom «Scarioth» (texte « occidental») / « Iscarioth»: (1) plusieurs racines hébraïques correspondent aux lettres sker ou sqr et signifient « mentir, trahir, tromper ; corrompre par de l'argent ; bouillonner »; (2) avec le $i$-initial, le surnom représente l'hébreu '-y-sh q-r-y-wt, « homme (des) villes », et apporte au «traitre » le visage contrasté du prédicateur urbain qu'a été Paul après sa conversion.
} 
disciples se donnent bientôt une base de prédication, à savoir la collection des paroles de Jésus, dont la rédaction est confiée à un certain Matthieu. Une autre allusion à cette décision initiale se trouve en Actes 1,23-26, avec le choix par le sort du successeur de Judas, entre Joseph Barnabas Justus et Matthias. Le premier représente la famille de Jésus (Joseph est le nom d'un de ses frères, en Mc 6,3 ou Mt 13,55) et le second porte le nom hellénisé du scribe qui rédigea pour les disciples la collection de paroles.

5) La tempête apaisée. La tempête est une image usuelle du conflit doctrinal (Eph 4,14) et du doute (Jc 1,5) : elle fait donc allusion, dans le contexte de cette tradition, à une dissidence, sans doute celle des Hellénistes (Ac 6 ), qui se produit très tôt dans la communauté primitive.

6) Exorcisme d'un démoniaque. Plusieurs indices dans l'épisode concourent à faire de la scène une métaphore de la conversion de Paul (Ac 9) : en particulier, le "pays des Géraséniens » (v. 1) fait référence à la ville de Gérasa, éloignée de la Mer de Galilée, mais étape incontournable sur le chemin de Damas depuis Jérusalem. L'esprit impur porte le nom de «Légion » (v. 9) et fait, au premier sens, allusion à l'armée romaine, ce qui n'apporte rien au sens; mais le mot est expliqué par le nombre : "car nous sommes plusieurs (ou nombreux) », ce qui caractérise les maittres de l'école pharisienne qu'a fréquentée Paul à Jérusalem. Après l'exorcisme, Jésus ne laisse pas le miraculé se joindre aux disciples, mais il l'envoie témoigner chez les siens, et celui-ci se rend dans la Décapole, réunion de dix villes, comme sont dix les principales étapes de Paul dans ses voyages missionnaires autour de la Mer Egée.

7) Guérison de deux femmes. Juste après l'exorcisme du démoniaque, Jésus est prié par un notable de venir guérir sa fille; en chemin, une femme touche son vêtement et obtient par ce geste la guérison de son mal ; Jésus parvient à la maison du notable et guérit sa fille. En Ac 9, après la conversion de Paul, Pierre opère deux guérisons, celle d'un homme portant le nom latin d'Enée et celle d'une sympathisante juive du nom de Tabitha. Dans ces deux passages à deux guérisons liées, la préséance de la guérison ne revient pas à celui / celle qui porte un nom juif : c'est le signe que la prédication des disciples à la tête de la communauté primitive se tourne désormais prioritairement vers le monde païen de l'empire romain. Dans le texte « occidental» de Marc, le lien entre les deux passages est renforcé par le fait que Jésus guérit la fille du notable en prononçant le nom de "Tabitha » au lieu de "Talitha ", dans les autres types de texte. L'épisode de Marc évoque donc la nouvelle priorité de s'adresser aux païens, donc aux gens de langue grecque.

8) Jésus dans sa patrie. Selon Ac 12, la direction de la communauté primitive change de mains au moment du règne d'Agrippa $1^{\text {er }}$, roi de Judée et de quelques autres régions de 41-44, par réunion 
des tétrarchies de ses oncles, désormais tous morts ${ }^{10}$. Jacques, le frère de Jésus, succède alors à Pierre. Or, dans l'épisode de Marc, quatre frères de Jésus sont nommés, avec Jacques en tête, en même temps qu'est soulignée la faible adhésion de la «patrie » de Jésus au nouveau message.

9) Envoi en mission des disciples. Les disciples, qui ne dirigent plus la communauté primitive, sont devenus des prédicateurs missionnaires, et les recommandations de Jésus font penser à celles que rédige Jacques, lors de la conférence évoquée en Ac 15 et Gal 2,110 : l'envoi en mission semble ainsi faire allusion à cette conférence, que l'on situe traditionnellement en 49.

10) Un incident en mission. L'un des disciples, Jean, atteste auprès de Jésus l'existence d'une prédication concurrente se réclamant également de Jésus, et Jésus légitime aussitôt cette autre prédication. Cet incident a un parallèle en Ac 19, quand Paul arrive à Ephèse et apporte un enseignement différent de celui d'Apollos déjà sur place. Le Jean de Marc correspond à Apollos, et l'enseignement concurrent, à celui de Paul; d'ailleurs, dans le texte «occidental» des Actes, Apollos est appelé «Apollonios» $(18,24)$, la deuxième partie du nom, après «Apoll-(os)» étant "Oni- », l'anagramme de Jean (Ioan-). Nous sommes donc à l'arrivée de Paul à Ephèse, au début de son deuxième voyage égéen, vers 56.

11) L'aumône de la veuve. Une image récurrente fait de la femme une communauté dont le chef serait le mari : la veuve est donc la métaphore d'une communauté qui vient de perdre son chef; et cette veuve, qui manifeste sa fidélité au temple par son aumône, est donnée en exemple par Jésus à ses disciples. Il n'en faut pas davantage pour voir dans cet épisode une allusion à la mort de Jacques, en 63, rapportée par Eusèbe (Hist. eccl. 2, 23), qui met la communauté primitive en situation précaire, au moment où son chef allait accéder à la fonction de grand-prêtre, autrement dit, la communauté était alors très proche du temple.

12) L'onction mortuaire de Béthanie. L'hommage que la femme rend à Jésus utilise le parfum ( $\mu \dot{\varrho} \varrho o v)$ employé pour l'embaumement du corps après la mort : le geste est donc l'image de la vénération par la communauté primitive de la mort de Jésus après celle de Jacques, qui est évoquée dans l'épisode précédent, et c'est cette vénération dont la mémoire est annoncée dans la phrase finale de l'épisode (v. 9), qui conclut tout l'ensemble narratif.

Le deuxième sens de ces douze épisodes est donc de proposer au lecteur instruit un survol de la première génération chrétienne, du ministère de Jésus à la mort de Jacques. Ce deuxième sens

10 Agrippa est le fils du fils aîné d'Hérode, Aristobule, mis à mort pour complot par son propre père. A la mort d'Hérode, le royaume est divisé en tétrarchies et le titre de roi n'est accordé à personne ; puis Agrippa obtient qu'il lui soit attribué en 41. Ainsi, au temps du ministère de Jésus, personne ne porte le titre de « roi des Juifs ». 
correspond parfaitement à ce qu'en dit Origène, dans son traité Des principes $(4,2,9)$, et Thomas d'Aquin l'atteste encore au XIII ${ }^{\mathrm{e}}$ siècle, quand il distingue du sens littéral le sensus rerum ou sens « historique », que P. Grelot explique ainsi : "Le sensus rerum est compris comme une réflexion théologique sur les événements historiques et tout ce qu'ils drainent dans leur cours : institutions, personnages, transformations des diverses composantes de la vie individuelle et sociale ${ }^{11} »$.

\section{Les compléments au "Marc romain »}

A ces douze épisodes communs à Marc et Luc, comprenant une réécriture et un déplacement pour deux d'entre eux chez Luc, s'ajoutent dans Marc une séquence narrative et plusieurs épisodes réunissant sept paroles de Jésus.

La séquence narrative se trouve en Mc 6,45-8,26; elle comprend cinq épisodes qui ont leur parallèle dans Matthieu (14,22-16,12), auxquels s'ajoutent deux guérisons absentes de Matthieu: celle d'un sourd, à la fin de l'épisode central (Mc 7,31-37), et celle d'un aveugle, à la fin de l'épisode final (Mc 8,22-26). Les cinq épisodes ont un deuxième sens qui est une nouvelle manière d'évoquer la première génération chrétienne : la marche sur les eaux $(6,45-56)$ est une image de la résurrection de Jésus, immatériel entre ciel et terre; le débat qui suit avec les pharisiens (7,1-23) comporte le signe de l'abandon de la loi rituelle et l'accent mis sur la loi morale, exprimée par la liste des douze péchés capitaux (v. 21-22) ; la rencontre de la Syro-phénicienne $(7,24-30)$ renvoie à l'ouverture de la prédication au monde païen ; la multiplication des pains $(8,1-$ 10) utilise des nombres qui font référence à une prédication ouverte à tous ${ }^{12}$; et la mise en garde finale $(8,11-21)$, réécrite lors de la rédaction finale pour faire la synthèse des deux multiplications des pains, disqualifie les enseignements du judaïsme en place, celui des Hérodiens, destiné à l'aristocratie, et celui des pharisiens, l'un et l'autre négligeant la prédication de l'espérance et du royaume de Dieu. A travers ces épisodes, on retrouve l'histoire en filigrane dans les douze épisodes précédents. Les deux guérisons qui s'y ajoutent traitent d'une question capitale qui se pose à la fin de la première génération : qui aura la légitimité, à la génération suivante ? La réponse associe celui qui reçoit l'enseignement par l'oreille (7,31-37), autrement dit le successeur des disciples et plus précisément de Simon Pierre ${ }^{13}$, et celui qui a reçu la connaissance

11 P. GReLot, Le langage symbolique dans la Bible, Initiations bibliques, Paris, 2001, p. 199.

${ }^{12}$ Le 4 de « 4000 » est l'image de l'homme en général, tandis que le nombre 5 de « $5000 »$, dans l'autre multiplication des pains qui fait partie de l'autre tradition narrative (commune aux trois synoptiques), est l'image de l'alliance, donc du peuple élu auquel Jésus s'adresse spécifiquement. 5 s'obtient, en effet, par l'addition de 4 (l'humanité) +1 (le monde divin) ou 2 (le monde terrestre) +3 (Dieu), évoquant ainsi l'alliance.

13 Rappelons que «Simon» est formé sur la racine $s h-m-$; « entendre »; et $\varepsilon \varphi \varphi \alpha \theta \alpha$, le mot que prononce Jésus au moment de la guérison, recouvre 
par la vision (8,22-26), à savoir Paul ; en somme, la légitimité est accordée à celui qui se réclamera à la fois de Pierre et Paul : ce sera le cas de Clément de Rome, puis d'Ignace d'Antioche.

Les sept paroles apparaissent par couples, pour six d'entre elles : d'abord l'homme fort et le péché contre l'esprit, terminant l'épisode 3,20-30, après une longue introduction sur le thème de l'unité nécessaire du peuple: ces paroles ont un deuxième sens politique et visent Hérode (l'homme fort) et son grand-prêtre (impardonnable); puis les paraboles de la semence qui pousse toute seule (4,26-29) et de la graine de moutarde (4,30-32), avant une conclusion commune où sont distingués le sens de l'image, destiné à la foule, et un deuxième sens expliqué aux disciples (4,33-34) : ce sont les deux axes de la prédication d'espérance, la lenteur de la venue du résultat et la merveille de celui-ci; vient ensuite la parole sur le plus grand, autrement dit le chef des chrétiens à la génération nouvelle, dans l'épisode 10,35-45 : c'est la parole centrale ; enfin, les deux paroles sur la foi à déplacer des montagnes, dans l'épisode 11,20-26, et sur l'amour du prochain, dans l'épisode 12,28-34: ces deux paroles symbolisent l'enseignement de Paul (voir 1 Co 13,2 et Lc 16,18) et celui des apôtres (voir Did 1,2 et Lc 10,27). On retrouve, par la disposition des paroles, la sructure formée par les régions, en Mc 3,7-8.

$\mathrm{Au}$ total, ces brèves indications permettent de conclure à l'unité que forment tous ces épisodes, venant d'une première tradition narrative et représentant le premier livre narratif chrétien écrit en deux temps par Marc, à la fois disciple de Paul $(\mathrm{Col} 4,10)$ et de Pierre (1 Pi 5,13).

\section{La structure septennale}

Aucun des épisodes de cet ensemble n'est explicitement daté ; mais leur disposition suggère une rupture avec le calendrier du temple et l'instauration d'un nouveau cycle des années.

\section{La rupture avec le temple}

Après le ministère de Jésus, contracté dans l'épisode de Capharnaüm $(1,21-39)$, et la mise en place d'une nouvelle communauté, avec une direction (3,31-35) et un écrit de référence $(4,21$ 25) dont les dates s'imposent et n'entrent dans aucun calendrier cyclique, survient un premier événement représenté par une tempête (4,35-41), à une date qui correspond à l'année sabbatique 32 ; dans la logique du temple, on attendrait un événement heureux, mais il s'agit de l'éclatement de la commnunauté primitive, peu de temps après sa naissance, avec la dissidence des Hellénistes, qui quittent bientôt Jérusalem pour se fixer, semble-t-il, à Alexandrie (Ac 8,22: "vers le midi»; 18,24-25: "Apollos, un Alexandrin, expert dans les Ecritures [la Septante] et formé dans sa patrie à la

l'hébreu $h-p-t-h$, « ouvre-toi », qui est un équivalent numérique de $p$-t-r en grec (Pierre) ou $k-y-p$ en araméen (Céphas). 
parole du Seigneur $\left.{ }^{14} »\right)$. Du point de vue du temple, il peut s'agir d'un événement heureux, puisque la communauté voit disparaitre un courant pratiquant le syncrétisme avec la sagesse grecque; mais pour la communauté, cette division est un affaiblissement: désormais, deux messages différents seront diffusés, se réclamant l'un et l'autre de Jésus. Ainsi se crée, peu de temps après la naissance de la communauté, une tension entre elle et le temple, du fait de la divergence de leurs intérêts. Du moins cette leçon peut être déduite de la manière de situer la tempête de la dissidence juste après la naissance de la communauté.

Un deuxième événement correspond à l'année sabbatique suivante, l'an 39, par la place qui lui est donnée, il est représenté par la guérison de deux femmes (5,21-43). En soi, la guérison est un événement heureux, mais l'accent de l'épisode porte sur l'ordre des guérisons: celle qui représente la foule passe en premier, s'insère dans le récit de l'autre, qui commence par une demande de guérison de la part d'un notable. Le renversement de l'ordre établi est un défi pour le temple : le thème en sera repris dans la deuxième tradition narrative, avec la priorité donnée aux enfants dans le royaume et la mise en garde faite aux riches pour l'accession à ce royaume. L'événement oppose à nouveau la communauté et le temple.

Une ancienne tradition, attestée par un écrit du IV siècle, la $S y$ nopse du Pseudo-Athanase, attribue à Jacques d'avoir traduit en grec la collection de paroles rédigée par Matthieu en araméen: elle est citée par Richard Simon, mais l'exégèse l'a ensuite oubliée. Or, Jacques devient le chef de la communauté sous Agrippa $1^{\text {er }}$, entre 41 et 44 : n'est-ce pas à cause de sa traduction qu'il est ainsi choisi, alors même que la famille a été reléguée, au moment de former la première direction de la communauté ? La traduction de Jacques serait donc de peu antérieure à 41, elle demande une fixation du vocabulaire grec qui rend compte de ce que les deux collections de Matthieu et de Luc, très différentes dans leur organisation, utilisent le même vocabulaire grec, qui s'oppose à celui de l'Evangile selon Thomas, partant du même modèle, mais pour en faire la base d'une autre idéologie. On voit bien, dans ces conditions, le rapport qui existe entre la traduction en grec des paroles de Jésus et la priorité donnée à la foule par la guérison de l'hémorroïsse: le grec est la langue de communication dans l'empire romain, tandis que l'araméen n'est parlé que dans une frange orientale de l'empire, celle qui jouxte l'empire perse dont la langue est un autre dialecte araméen (le syriaque), proche de celui parlé en Judée (le judéo-araméen), et a encore quelques locuteurs dans les synagogues de l'empire romain, où le grec est devenu la langue dominante. Passer de l'araméen au grec, c'est rendre les paroles de Jésus accessibles à l'ensemble du monde romain, c'est donner la priorité à la foule hellénophone sur l'élite juive qui

\footnotetext{
${ }^{14}$ D'après le texte «occidental»; le texte alexandrin ne mentionne pas la

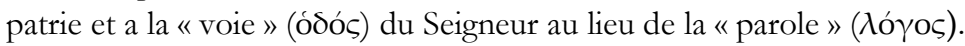


s'exprime traditionnellement en araméen. Et l'événement est confirmé dans les Actes (9,32-43), par l'ordre des guérisons d'Enée et de Tabitha et le choix de leurs noms.

L'événement marque la rupture avec le temple : il n'y aura pas d'autre fait correspondant à une année sabbatique. Les chrétiens ont désormais recours à un autre calendrier, dont le point de départ se situe entre ces deux années sabbatiques : il s'agit de la conversion de Paul.

\section{La construction d'un nouveau cycle}

La date de la conversion de Paul n'est pas connue avec précision, elle dépend de la manière de lire les données de Paul, au début de Galates : faut-il additionner les «trois ans » $(1,18)$ qui séparent la conversion et la rencontre de Pierre à Jérusalem ${ }^{15}$ et les «quatorze ans » $(2,1)$ qui précèdent la conférence de Jérusa$\operatorname{lem}^{16}$ ou inclure les trois ans au début de la période des quatorze? L'exégèse hésite ${ }^{17}$, M.-F. Baslez choisit la première solution ${ }^{18}$ et situe la conversion de Paul en 34, reculant la conférence de Jérusalem en 51, alors que la date consensuelle la situe en $49^{19}$. En réalité, les deux combinaisons sont à prendre en compte. L'enchaînement de la tempête (Mc 4,35-41) et de l'exorcisme du démoniaque $(5,1-20)$ suggère deux événements proches, ce qui conforte la date de 32 pour la conversion de Paul, soit dix-sept ans avant la conférence de 49 ; mais la nature heureuse de cette conversion amène à la transmettre comme si elle avait eu lieu en 35 , soit quatorze ans avant la conférence de 49 , pour fonder ainsi un nouveau cycle septennal qui va servir à structurer toute la première génération.

Par cette construction, l'an 35 devient le nouveau repère d'un cycle qui n'est plus sabbatique, avec les années 32 ou 39, mais dominical, avec comme premier événement heureux, la conversion de Paul. Et par ce transfert s'exprime à la fois la rupture avec le temple et le caractère funeste de la dissidence des Hellénistes, puisque 32 devient une quatrième année dans le nouveau cycle, une année funeste, comme le fut dans l'ancien cycle la prise de Jérusalem en -586 (voir plus haut). Dans ces conditions, la montée de Paul à Jérusalem, trois ans après sa conversion, est transposée de 35 à 38 et annonce ainsi le retournement qui privilégie la

${ }^{15} \mathrm{Gal} 1,18$ : «Ensuite, trois ans après, je montai à Jérusalem... »

${ }^{16}$ Gal 2,1 : «Ensuite, au bout de quatorze ans, je montai de nouveau à Jérusalem » : rien n'empêche de donner aux deux durées indiquées le même point de départ, la conversion de Paul.

${ }^{17}$ F. VOUGA, «Chronologie paulinienne », dans D. MArguerat (éd.), Introduction au NT, Genève, 2000, 2001², p. 131-138.

18 M.-F. Baslez, Saint Paul, artisan d'un monde chrétien, Paris, 1991, 2008², p. 441-445.

${ }^{19}$ Cette date est déterminée par la mort d'Agrippa $1^{\text {er }}$ en 44 (Ac 12,23) et la famine des années 46-48 (Ac 11,28). 
foule au détriment de l'élite juive et de ses alliés - sans doute cela vise-t-il les pharisiens. L'année 39 est, en somme, le dernier lien avec le cycle sabbatique du temple, elle est marquée par le retournement qui privilégie le monde païen sur le peuple juif, la foule sur l'élite et correspond sans doute à la traduction en grec par Jacques de la première collection de paroles rédigée en araméen.

Le nouveau repère de l'an 35 fait de l'année 28, où Jésus reçoit le baptême et commence son ministère, le point de départ du nouveau cycle dominical, le début de l'histoire du christianisme. Cette fonction de commencement explique la solennité avec laquelle, plus tard, la rédaction finale de Luc datera le baptême de Jésus (Lc 3,1-2), en confirmant la date de 28.

Après 35, les années dominicales tombent en 42, 49, 56 et 63, l'auteur écrivant au cours du cycle suivant, avant 70 . Or, les quatre épisodes venant après la guérison des deux femmes (5,21-43) correspondent à ces années : l'échec de Jésus dans sa patrie (6,1-6), contenant la liste de ses frères, à l'année 42, c'est-à-dire au début du mandat de Jacques à la tête de la communauté primitive ; l'envoi en mission (6,7-13), à la conférence de Jérusalem de 49, qui étend la prédication missionnaire aux terres lointaines, en faisant référence à la seule loi noachique ${ }^{20}$; l'incident en mission (9,38-50), à l'arrivée de Paul à Ephèse, en 56, où il développe la christologie ; et la pauvre veuve qui manifeste sa fidélité au temple malgré la perte de son mari (12,41-44), à la mort de Jacques en 63, sur le point de devenir le grand-prêtre du temple de Jérusalem.

La trame du « Marc romain » est, en somme, la base d'un nouveau calendrier cyclique, où les années sont toujours comptées par sept, mais où l'année dominicale de référence est décalée par rapport aux années sabbatiques du calendrier du temple. Et l'on peut lire dans ce décalage un double message : d'une part, la volonté de rompre avec le sacerdoce hérodien, où le grand-prêtre est nommé par l'autorité civile et non par Dieu, ce qui en fait un usurpateur; et d'autre part, l'ambition d'installer au temple un grand-prêtre qui serait le successeur de Jésus, ou plus exactement, du fait de sa résurrection, son lieutenant se laissant inspirer par lui, devenu céleste, et exerçant la fonction suprême au temple avec la pleine légitimité divine. Le souci de l'auteur de ce livret n'est pas tant de rappeler quelques faits marquants de la vie de Jésus que d'utiliser l'histoire de la première génération chrétienne pour fonder une nouvelle lecture cyclique du temps, alors que le temple existe encore et que les chrétiens espèrent y installer l'un des leurs.

20 Sur la complexité du «décret apostolique» transmis sous plusieurs formes textuelles en Ac 15,20.29, voir C.-B. AMPHOUX, "Les variantes et l'histoire du 'décret apostolique'", dans A. DenAux, NT Texual Criticism and Exegesis, Mél. J. Delobel, BETL 161, Leuven, 2002, p. 209-226. Le terme de loi noachique fait référence à Gn 9,1-17, où Dieu s'adresse à Noé, seul rescapé du déluge, donc le père de la nouvelle humanité. 


\section{L'abandon du nouveau cycle}

Le «Marc romain » ne relate pas des moments du ministère de Jésus, mais il met en scène l'abandon par la première génération chrétienne du cycle sabbatique en usage au temple de Jérusalem et la mise en place d'un nouveau cycle qui traduit la volonté d'autonomie des chrétiens, par rapport au calendrier du temple, et l'ambition de mettre en place une réforme qui modifie ce calendrier. Nous plongeons ainsi dans un premier christianisme dont nous avons perdu la mémoire et qui va prendre fin en 70 , avec la destruction du temple.

Après 70, en effet, on ne trouve plus trace de ce calendrier. La deuxième tradition narrative se concentre sur le ministère de Jésus, elle développe le contenu du premier épisode du «Marc romain » sans envisager l'après-Jésus : il faudra adjoindre à l'évangile le livre des Actes, pour couvrir la période suivante. Et l'on serait en peine de dégager de ce livre un cycle dominical pour la période suivante, puisque le récit s'arrête à l'arrivée de Paul à Rome, avant la mort de Jacques. Rien n'indique non plus chez les historiens la survivance de ce cycle, que l'on observe dans le "Marc romain ». La perspective des chrétiens n'est plus d'instaurer un calendrier, mais de fonder une espérance sur la mort rédemptrice de Jésus, qui prend désormais place à la fin de l'évangile avec le sens que lui donne Paul, quand il est à Ephèse, en 56 et 57. Le cycle dominical de la première génération chrétienne tombe dans $l^{\prime}$ oubli ${ }^{21}:$ la rédaction finale fera des récits conçus pour marquer les années dominicales des épisodes du ministère de Jésus. Le temps est compté, désormais, selon la chronologie royale (ou impériale), qui n'est plus cyclique, mais linéaire. On ira jusqu'à perdre de vue que la Bible juive compte le temps de deux façons à la fois, selon la chronologie royale et selon le calendrier septennal du temple.

\section{Conclusion}

Loin d'être un livre simple et d'un seul tenant, l'évangile selon Marc présente une grande complexité, reposant sur la réunion de deux traditions narratives écrites l'une et l'autre dans la culture judéo-hellénistique, qui est une culture savante, née au $\mathrm{III}^{\mathrm{e}}$ siècle avant notre ère de la rencontre de la tradition juive et de la culture grecque d'Alexandrie. Cette culture est notamment caractérisée par la pratique d'une écriture à deux niveaux de sens, celui apparent qui est destiné à la foule et sur lequel repose la prédication, et le sens profond qui est réservé à une élite cultivée, à laquelle il est transmis par l'explication, puisqu'il n'apparaît pas à la lecture ; mais le lecteur attentif en devine l'existence, en observant les discordances et les aspérités du récit. Par ce sens, l'auteur transmet un enseignement plus approfondi, qui arme notamment celui qui

\footnotetext{
${ }^{21}$ Ce cycle se transmet malgré tout par l'organisation de la semaine et le décalage du jour du repos du septième jour (le shabbat) au premier (le dimanche ou jour dominical).
} 
le reçoit pour la prédication, à la manière dont la science médicale arme le médecin pour soigner les malades.

La première tradition narrative qui constitue Marc est écrite dans un premier temps à Rome entre la mort de Jacques à Jérusalem en 63 et celle de Pierre à Rome en 64, puis elle est amplifiée dans un deuxième temps à Alexandrie entre la mort de Pierre et la destruction du temple en 70 . La première rédaction, que nous avons appelé le "Marc romain », comprend douze épisodes de longueur variable, allant de quatre ou cinq versets pour les plus courts $(3,31-35 ; 4,21-25 ; 12,41-44)$ à une vingtaine pour les plus longs $(1,21-39 ; 5,1-20 ; 5,21-43)$. Et sept de ces douze épisodes signalent un abandon progressif du calendrier cyclique du temple au profit d'un nouveau calendrier également cyclique, qui se fonde sur la conversion de Paul, transposée en 35. La rupture avec le temple et l'abandon de son calendrier coïncident avec le moment où la prédication privilégie le contact avec la foule de langue grecque, c'est-à-dire le monde païen de l'empire romain ; et l'on peut dater la décision de ce tournant de l'an 39, peu après la première venue de Paul à Jérusalem, qui apparait ainsi comme à l'origine de la décision. Paul mentionne cette montée à Rome (Gal 1,18) ainsi que les Actes $(9,26-29)$, qui situent juste après la guérison d'Enée par Pierre, avant celle de Tabitha, et donne ainsi la préséance au païen.

Dès lors, la conversion de Paul apparaît comme la réponse divine à la crise provoquée par la dissidence des Hellénistes, et elle est associée à l'année qui va servir de fondement au nouveau cycle dominical que les chrétiens veulent mettre en place, à savoir l'an 35 , soit sept ans après le début du ministère de Jésus. Et quatre événements vont compléter la conversion de Paul pour créer la structure septennale de la première génération chrétienne: l'installation de Jacques et des frères de Jésus à la tête de la communauté, en remplacement des apôtres, en 42 ; la conférence de Jérusalem sous l'autorité de Jacques, avec la présence de Paul comme délégué de l'Eglise d'Antioche, en 49 ; la rencontre entre l'enseignement de Paul et celui de Jean à Ephèse, en 56 ; et la mort de Jacques à Jérusalem, en 63. Entre les épisodes qui rappellent ces événements, aucun autre ne s'insère pour correspondre aux années sabbatiques: le calendrier du temple est désormais remplacé. En 56, quand Paul est à Ephèse, il propose de substituer au shabbat le premier jour de la semaine, qui devient celui de la commémoration de la résurrection, le dimanche. Le calendrier dominical qui se met en place sera abandonné arès 70 ; mais le changement du jour du repos hebdomadaire sera conservé et deviendra la cractéristique de la semaine chrétienne. 